\title{
Multi-Satellite Cycle-Slip Detection and Exclusion using the Noise Subspace of Residual Dynamics
}

\author{
Jaume Riba $^{\dagger}$, Senior Member, IEEE, Ferran De Cabrera ${ }^{\dagger}$, Student Member, IEEE and José-Miguel Juan ${ }^{\ddagger}$ \\ ${ }^{\dagger}$ Signal Theory and Communications Department, Technical University of Catalonia (SPCOM/UPC) \\ ${ }^{\ddagger}$ Research Group of Astronomy and Geomatics (gAGE/UPC) \\ \{jaume.riba, ferran.de.cabrera, jose.miguel.juan\}@upc.edu
}

\begin{abstract}
Real-time detection of cycle-slips on undifferenced carrier-phase measurements is an important task to properly exclude wrong phase trackers from precise positioning algorithms. The detection is especially challenging in high-dynamic mobile scenarios, where traditional approaches (as those based on single-channel polynomial fitting) may easily lead to false positives. Using a multi-channel formulation of the problem, the proposed technique takes benefit of the available data redundancy (high number of tracked satellites) in order to ameliorate the false positives. This robustness is accomplished by adaptively estimating the orthogonal subspace spanned by the polynomial time-varying residuals obtained from all available channels (treated as a vector process), and using that subspace to form efficient channel combinations with cancelled satellitereceiver dynamics. The main advantage of the multi-channel approach is that wrong measurements can be discarded without needing any positioning estimate nor phase-ambiguity solver, thus improving the accuracy, reliability and integrity of positioning. The performance improvement is shown by means of theoretical analysis and computer simulations.
\end{abstract}

Index Terms-Cycle-Slips, SVD, MIMO, GLRT, GNSS.

\section{INTRODUCTION}

Undifferenced carrier-phase measurements are used in highaccuracy navigation systems, such as the GPS Precise Point Positioning (PPP) [1], for stand-alone geodetic point positioning (static or kinematic) with centimeter precision. In PPP, although most of the ionospheric delay is cancelled by means of the dual-frequency ionosphere-free combination [1], a long convergence time for estimating the phase ambiguities is required before providing accurate navigation solutions [2]. In practice, carrier-phase measurements suffer from anomalies such as cycle-slips (caused by poor signal to noise ratio, signal blockage or ionospheric scintillation), receiver clock jumps and satellite-receiver dynamics, among others. As cycle-slips have a persistent and accumulative nature, reduction in positioning accuracy and loss of integrity ensues [3] unless they are repaired or otherwise dealt with. Although high precision can also be obtained from only raw code measurements through singular spectrum based smoothing [4], cycle-slip free carrier phases from classical closed-loops are normally preferred in dual-frequency receivers, even in open loops [5].

Several methods have been proposed in the past for cycleslip detection (see [6], [7] and references therein). A common

This work has been supported by projects TEC2016-76409-C2-1-R (WINTER), Ministerio de Economia y Competividad, Spanish National Research Plan, and 2017 SGR 578 - AGAUR, Catalan Government. We thank Dr. Adriano Camps (UPC) and ESA project 1-8214/15/NL/LvH (SCIONAV). approach to enhance detection reliability is to form combinations of measurements to cancel out the time-varying geometric range caused by satellite-receiver dynamics and receiver clock instabilities. While satellite dynamics can be mostly cancelled by de-trending (for example, through timedifferentiating the carrier-phase measurements), receiver dynamics is a much less systematic phenomenon that requires special combinations to cope with [8][1]. For example, the dual-band geometry-free combination cancels the satellitereceiver dynamics, but it is affected by a time-varying ionospheric refraction term and scintillation. Another example is the Melbourne-Wübbena combination [9], which is both geometry and ionosphere-free, but it is notably noisier, as code measurements are mixed with carrier-phases. In both cases, the added noise effects make the detection mechanisms more prone to false positives.

The structure and main aspects of this paper are summarised in the sequel. In Section II we formulate the problem on a multi-channel basis. In Section III we give an original synthesis of the polynomial fitting idea [10] and high-order time-differentiating approaches [8] for step-detection, which are cast as a quadratic minimization problem with linear constraints. This formulation allows to highlight the trade-off between noise enhancement and latency in the single-channel detection stage. Then, the core subspace idea in "satellite"domain is proposed in Section IV, which aims to detect and exclude cycle-slips in high-dynamic receiver conditions by simultaneously processing and adaptively combining the polynomial residuals of carrier-phase measurements obtained from multiple satellites, and without the need of having receiver position estimated nor carrier-phase ambiguities fixed. The proposed technique makes use of the fact that receiver dynamics residuals, along with receiver clock instabilities, are confined on a subspace of the covariance matrix estimated from the multi-channel vector residuals. Therefore, eigenvectors associated to the smallest eigenvalues of that covariance matrix, which span the so-called noise (or minor) subspace, provide optimal combinations of measurements for reliable cycle-slip detection. With respect to the approach presented in [6], which uses the singular spectrum of windowed carrier phase measurements to detect anomalies in single-channel mode, the main novelty of the present formulation lies on the usage of the noise subspace of the overall redundant multi-channel data, which allows the formulation of widelyused design criteria for composite hypothesis testing. For 
concreteness, we develop the Generalized Likelihood Ratio Test (GLRT) [11] associated to the formulated problem, while other detection criteria such as Rao and Wald tests are left for future consideration. The main motivation behind the overall formulation is the fact that tracking the noise subspace is potentially much less demanding (in terms of required precision and speed of adaptation) than tracking the receiver position. An additional motivation is to obtain a low complexity detection scheme, totally uncoupled with any estimate of receiver position (in contrast with [12] and the integrated adjustment proposed in [13]) and with any navigation data received from satellites. Section IV also provides a novel method to identify and exclude the anomalous measure (i.e. identifying the fault channel) exploiting the subspace structure, which is based on non-coherent communications theory. Finally, simulation results to highlight the benefits of the proposed method are given in Section $\mathrm{V}$, and main conclusions drawn in Section VI.

Notation: $(\cdot)^{H}$ denotes the Hermitian operator, $|\cdot|$ the modulus, $\|\cdot\|$ the euclidean norm, $[\cdot]_{i}$ the $i$-th entry of a vector, $[\cdot]_{i, j}$ the entry at $i$-th row and $j$-th column of a matrix, $\odot$ the Schur-Hadamard (element-wise) product between two vectors, $R e[\cdot]$ the real part, $\operatorname{diag}(\cdot)$ the main diagonal of a square matrix, $(\cdot)^{\alpha}$ a vector with elements $\left[(\cdot)^{\alpha}\right]_{i}=\left([\cdot]_{i}\right)^{\alpha}, \mathbf{0}$ the all-zeros column vector, and $\mathbf{1}$ the all-ones column vector.

\section{PROBLEM FORMULATION}

Let $S$ be the number of channels (i.e. the available number of tracked satellites), $n$ the integer time index associated to the sampled measurements, and $K$ the observed time-window size. The $K \times S$ run-time data matrix is defined as

$$
[\mathbf{Y}(n)]_{k, s}=\varphi_{s}^{I F}(n-k+1)
$$

with $1 \leq s \leq S$ and $1 \leq k \leq K$, where $\varphi_{s}^{I F}(n)$ is the GNSS measurement sequence associated to $s$-th satellite, estimated using the dual-carrier ionosphere-free $(I F)$ combination to achieve efficient ionospheric residual and scintillation cancellation [1]. As the measurements are derived from carrier phases, they have an inherent ambiguity (given by multiples of the wave-lengths) that has to be solved before they can be used for precise positioning. If cycle-slips were produced after fixing ambiguities without being detected (and the corresponding wrong channel excluded), the resulting accumulated ambiguity would yield a negative impact on positioning accuracy.

In absence of cycle-slips, the non-anomalous data matrix is modeled by four additive contributions:

$$
\mathbf{Y}(n)=\mathbf{X}^{H}(n) \mathbf{G}^{H}(n)+\mathbf{t}(n) \mathbf{1}^{H}+\mathbf{H}_{L} \mathbf{P}(n)+\mathbf{W}(n)
$$

It is noted that the Hermitian (instead of Transpose) operator is used in all equations for generality. The reason is that, although the original data is real, complex filtering will be allowed for the reasons that will become clear in Section IV.

The rationale of the model in Eq. (2) is as follows:

- The first contribution is based on the traditional linearized range model, where the $3 \times K$ receiver-dynamics matrix $\mathbf{X}(n)$ contains the (possibly time-varying) $x, y, z$ receiver-position components at every column, and the rows of the $S \times 3$ (time-varying) geometry-matrix $\mathbf{G}(n)$ contain the (unknown) unitary line-of-sight vectors from receiver to every satellite.

- The second contribution models the receiver-clock jitter, where $\mathbf{t}(n)$ is the $K \times 1$ run-time vector of the receiver clock error sequence, a common phenomenon that is identically mapped in all measurements.

- The third contribution assumes that other slow varying effects, such as the satellite dynamics or tropospheric delays, fit a polynomial of order $L$ inside the observation window, where $\mathbf{H}_{L}$ is a $K \times(L+1)$ Vandermonde matrix whose elements are $\left[\mathbf{H}_{L}\right]_{k, l}=k^{l-1}$, with $1 \leq k \leq K$ and $1 \leq l \leq L+1$, and $(L+1) \times S$ matrix $\mathbf{P}(n)$ contains the (unknown) polynomial regression coefficients per satellite in their columns.

- Finally, the fourth contribution (with $[\mathbf{W}(n)]_{k, s}=$ $\left.w_{s}(n-k+1)\right)$ denotes the multi-channel measurement error (see [1] for details), where $w_{s}(n)$ is Gaussian i.i.d. measurement white noise with variance $\sigma^{2}$.

The uncoupling assumption with respect to prior position estimates implies that matrices $\mathbf{X}(n)$ and $\mathbf{G}(n)$ in Eq. (2) are assumed unknown.

Let us define the multi-channel data vectors $\mathbf{y}_{d}(n)(S \times 1)$ and $\mathbf{y}_{d c}(n)((S-1) \times 1)$ as:

$$
\begin{aligned}
\mathbf{y}_{d}(n) & =\mathbf{Y}^{H}(n) \mathbf{h}_{L} \\
\mathbf{y}_{d c}(n) & =\mathbf{V}^{H} \mathbf{y}_{d}(n)
\end{aligned}
$$

where the $K \times 1$ time-filter $\mathbf{h}_{L}$ and $S \times(S-1)$ spatial-filter $\mathbf{V}$ are designed to cancel-out the third and second terms in Eq. (2), respectively. As a result of the temporal and spatial filtering formulated in Eqs. (3) and (4), respectively, in nonkinematic mode, the components of $\mathbf{y}_{d c}(n)$ will contain only the residuals of every satellite associated to step-anomalies and measurement noise. However, in kinematic mode, the receiverdynamics (first term of Eq. (2)) will be still prone to mask these anomalies and, as a consequence, produce false positives. Ameliorating these false positives is the main purpose of the multi-channel method proposed in Section IV.

\section{A. Summary of the main contribution}

As the geometry matrix $\mathbf{G}(n)$ in Eq. (2) is of rank-3, and one degree of freedom has been already lost through the spatial filtering in Eq. (4), the key idea to reliably detect and exclude step-anomalies without the need of estimating $\mathbf{X}(n)$ (but still being robust to high receiver dynamics) is to exploit the resultant eigen-structure of the $(S-1) \times(S-1)$ multi-channel covariance matrix of the vector process $\mathbf{y}_{d c}(n)$ containing the polynomial fitting residuals from all channels, estimated from the past $N$ samples as

$$
\hat{\mathbf{R}}_{\mathbf{y}_{d c}}=\frac{1}{N} \sum_{m=K}^{K+N-1} \mathbf{y}_{d c}(n-m) \mathbf{y}_{d c}^{H}(n-m)
$$

where $K$ is some estimation latency, equal to the latency of single-channel anomaly detectors described in the sequel. This assures that the eigen-structure of $\hat{\mathbf{R}}_{\mathbf{y}_{d c}}$, exploited later on to cancel high dynamics, will not be affected by the own anomalies that we want to detect. 


\section{Single-CHANNEL POLYNOMIAL FITTING}

The de-trending fixed filter $\mathbf{h}_{L}$ in Eq. (3) can be designed in different ways. Some well-known designs include the successive phase differentiation approach (see [8] and references therein) with $K=L+2$. For example, for $L=3$, the filter coefficients would be trivially designed as $\mathbf{h}_{3}^{H}=[1,-4,6,-4,1] / 16$ whose squared step response (given by $[1,9,9,1] / 256$ ) would provide a statistic for a simple step-detection test. Similar models have been used in [14].

To avoid the noise enhancement phenomenon associated with differentiating, a more general filter design is proposed with (odd integer) $K>L+2$ such that the extra degrees of freedom are used to yield minimum noise enhancement and maximum unite-step response at the middle point of the observation window. This problem can be cast as a quadratic minimization scheme with $L+2$ linear constraints (designed to cancel the third term in Eq. (2)) as follows:

$$
\operatorname{Min}_{\mathbf{h}_{L}}\left\|\mathbf{h}_{L}\right\|^{2} \text { subject to } \mathbf{H}_{L}^{H} \mathbf{h}_{L}=\mathbf{0} \& \mathbf{b}^{H} \mathbf{h}_{L}=1
$$

with the following rationale: i) minimizing $\left\|\mathbf{h}_{L}\right\|^{2}$ provides minimum impact of the measurement noise on detection performance; ii) the first constraint aims at cancelling the polynomial trend; and iii) the second constraint aims at providing a non-zero sensitivity to a step, where $\mathbf{b}$ is a $K \times 1$ vector modeling samples of a centered unite-step, that is, $[\mathbf{b}]_{k}=\operatorname{sign}(k-(K-1) / 2) / 2$.

Using Lagrange multipliers, the solution of Eq. (6) becomes:

$$
\mathbf{h}_{L}=\tilde{\mathbf{H}}_{L} \mathbf{f}_{L}
$$

where $\tilde{\mathbf{H}}_{L} \doteq\left[\mathbf{b}, \mathbf{H}_{L}\right]$ is the $K \times(L+2)$ extended constraint matrix and $\mathbf{f}_{L}$ is the first column of matrix $\mathbf{F}_{L}=$ $\left(\tilde{\mathbf{H}}_{L}^{H} \tilde{\mathbf{H}}_{L}\right)^{-1}$. Note that, for $L$ even, as $\mathbf{h}_{L}$ inherits the odd symmetry of $\mathbf{b}$, the constraint $\mathbf{H}_{L}^{T} \mathbf{h}_{L}=\mathbf{0}$ implies $\mathbf{H}_{L+1}^{T} \mathbf{h}_{L}=\mathbf{0}$ and, therefore, $\mathbf{h}_{L+1}=\mathbf{h}_{L}$, for which only even values of $L$ are of interest.

An insightful indicator of the unite-step detection performance is the gain in signal-to-noise ratio (also known as deflection in detection theory) at the de-trending filter output, which is easily given by

$$
G_{S N R}=\left(\left|\mathbf{b}^{H} \mathbf{h}_{L}\right| /\left\|\mathbf{h}_{L}\right\|\right)^{2}=\left[\mathbf{F}_{L}\right]_{1,1}^{-1}
$$

It can be shown that designing the step at the middle of the window maximizes $G_{S N R}$ and makes sequence $\left[\mathbf{F}_{2 l}\right]_{1,1}^{-1}$ monotonically decreasing with $l$, with $\left[\mathbf{F}_{0}\right]_{1,1}^{-1}=K / 4$.

Fig. (1) shows $G_{S N R}$ as a function of $K$ for different orders $L$, comparing with the traditional phase-differentiation approach. Clearly, increasing $K$ for a fixed $L$ allows improving $G_{S N R}$ at the expense of a longer detection latency of roughly $K / 2$ samples, as the sensitivity to a discontinuity is maximized at the middle of the observation window.

\section{Multi-Channel Dynamics CANCELLation AND CYCLE-SLIP DETECTION}

The proposed detection scheme consists on the following three stages:

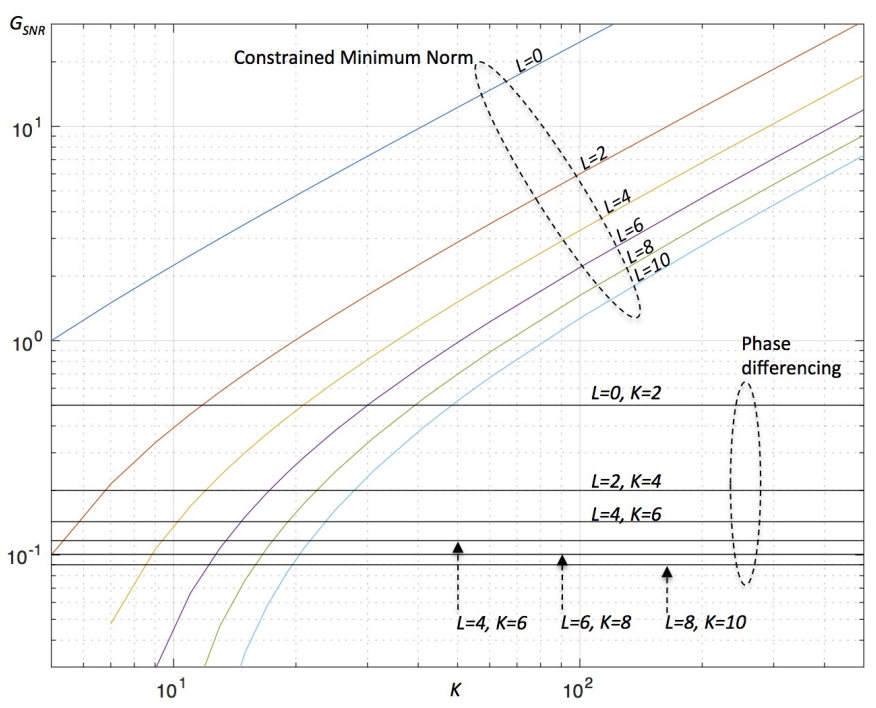

Fig. 1. SNR gain of the minimum norm criterion vs. $K$ for different $L$.

a) Deterministic clock subspace cancellation: Matrix V in Eq. (4), which can be computed off-line, should fulfill $\mathbf{V}^{T} \mathbf{1}_{S}=\mathbf{0}$ to cancel the second term in Eq. (2). A simple design ([8][1]) is based on forming differences from pairs of satellites, such that a single column of $\mathbf{V}$ has only two non-zero entries with values 1 and -1 . This procedure is equivalent to forming Time-Difference-of-Arrivals (TDOA) based on carrier-phases, as it is used in hyperbolic positioning. This design, however, is discarded because it leads to correlated noise after the combination, which would induce a dimension-dependent Mahalanobis norm and distance (instead of Euclidean) in the GLRT proposed later on (see Eq. (13)). For that reason, unitarity of $\mathbf{V}$ is preferred. To be concrete, $\mathrm{V}$ is constructed from the $S \times S$ orthonormal Fourier matrix after removing the column associated with the zero-frequency beam, which yields uncorrelated noise components on $\mathbf{y}_{d c}(n)$. Note that complex data is then obtained on $\mathbf{y}_{d c}(n)$.

b) Block adaptive receiver-dynamics subspace cancellation: Let $\mathbf{U}$ be a $(S-1) \times(S-4)$ unitary matrix spanning the noise subspace of the de-trended data covariance matrix $\hat{\mathbf{R}}_{\mathbf{y}_{d c}}$ given in Eq. (5). For $S \geq 5$, matrix $\mathbf{U}$ can be obtained adaptively either from $\hat{\mathbf{R}}_{\mathbf{y}_{d c}}$ through SVD in block processing, or from the direct data $\mathbf{y}_{d c}(n)$ in continuous processing using, for instance, the well-known efficient and robust orthogonal Oja (OOja) algorithm described in [15], which, in addition, ensures the orthonormality of $\mathbf{U}$ at each iteration. The spatial filter consists in projecting the de-trended data vector defined in Eq. (4) onto the noise subspace as follows:

$$
\mathbf{z}(n)=\mathbf{U}^{H} \mathbf{y}_{d c}(n)=\mathbf{C}^{H} \mathbf{y}_{d}(n)
$$

where

$$
\mathbf{C}=\mathbf{V U}
$$

is a $S \times(S-4)$ matrix that represents the overall MIMO channel affecting the anomalies in $\mathbf{y}_{d}(n)$. As a result, in the obtained $(S-4) \times 1$ vector sequence $\mathbf{z}(n)$, satellite trending, receiver dynamics and receiver clock instabilities are totally cancelled. In fact, $\mathbf{z}(n)$ contains only the effect of the additive 
measurement noise and the effect of potential step-anomalies produced in some channel.

c) Multi-channel detection and exclusion: Splitting Eq. (9) into the anomaly and noise contributions yields:

$$
\mathbf{z}(n)=\beta \mathbf{C}^{H} \mathbf{e}(n)+\mathbf{w}(n)
$$

where $\mathbf{e}(n)=\mathbf{0}$ in absence of anomalies (hypothesis $\mathcal{H}_{0}$ ). When a single step-anomaly is present in one channel (hypothesis $\mathcal{H}_{1}$ ), as a consequence of the peaky de-trending filter unite-step response, vector $\mathbf{e}(n)$ is modelled as $\mathbf{e}(n) \in$ $\left\{\mathbf{e}_{s}\right\}_{s=1, \ldots, S}$, where $\left\{\mathbf{e}_{s}\right\}$ is the canonical orthonormal basis ( $\mathbf{e}_{s}$ has a single 1 in its entry $s$, the index of the fault satellite). As a result of the imposed unitarity on $\mathbf{V}$ and $\mathbf{U}$ (and therefore on $\mathbf{C}$ ), the noise term $\mathbf{w}(n)$ has a diagonal covariance matrix determined by the de-trending filter, that is

$$
\mathbf{R}_{\mathbf{w}}=E\left[\mathbf{w}(n) \mathbf{w}^{H}(n)\right]=\left(\sigma^{2} / G_{S N R}\right) \mathbf{I}
$$

where $G_{S N R}$ is given in Eq. (8). Therefore, the problem of anomaly detection can be cast as an equivalent problem of noncoherent detection in orthogonal transmission in uncorrelated Gaussian noise in the presence of a partially-known complex MIMO channel $\mathbf{C}$ (estimated from the own data), and an unknown real multiplicative fading term $\beta$. Since hypothesis $\mathcal{H}_{1}$ has unknown parameters (real $\beta$ and integer $s$ ), a GLRT is proposed for step-anomaly detection, which, under the assumption that $\mathbf{w}(n)$ has i.i.d. normal entries as shown in Eq. (12), easily leads to minimizing the difference between Euclidean norm $\|\mathbf{z}(n)\|^{2}$ and distance $\left\|\mathbf{z}(n)-\beta \mathbf{c}_{s}\right\|^{2}$ against $s$ and $\beta$ or, equivalently,

$$
T(n)=\max _{s, \beta}\left(2 \beta R e\left[\mathbf{c}_{s}^{H} \mathbf{z}(n)\right]-\beta^{2}\left\|\mathbf{c}_{s}\right\|^{2}\right)
$$

where $\mathbf{c}_{s}=\mathbf{C}^{H} \mathbf{e}_{s}$, such that an anomaly is declared if $T(n)>$ $\gamma$, with $\gamma$ some threshold. Note that GLRT naturally provides Maximum Likelihood estimates of $s$ (index identifying the fault channel) and $\beta$ (the anomaly step-size). Optimizing first for $\beta$ for a given $s$ yields $\hat{\beta}=\left\|\mathbf{c}_{s}\right\|^{-2} \operatorname{Re}\left[\mathbf{c}_{s}^{H} \mathbf{z}(n)\right]$, which re-inserted into the original test in Eq. (13) yields,

$$
T(n)=\max _{s}\left(\frac{\operatorname{Re}\left[\mathbf{c}_{s}^{H} \mathbf{z}(n)\right]}{\left\|\mathbf{c}_{s}\right\|}\right)^{2}
$$

As $\mathbf{c}_{s}^{H}$ is the $s$-th row of MIMO matrix $\mathbf{C}$, we can define:

$$
\begin{gathered}
\mathbf{d}=\operatorname{diag}\left(\mathbf{C C}^{H}\right) \\
\mathbf{t}(n)=\mathbf{d}^{-1 / 2} \odot \operatorname{Re}(\mathbf{C z}(n))
\end{gathered}
$$

Then, a more compact expression of the final detector can be written as follows:

$$
T(n)=\max _{s}\left([\mathbf{t}(n)]_{s}\right)^{2}
$$

and the fault channel index and step estimates are given by:

$$
\begin{gathered}
\hat{s}=\arg \max _{s}\left([\mathbf{t}(\hat{n})]_{s}\right)^{2} \\
\hat{\beta}=\left[\mathbf{d}^{-1 / 2} \odot \mathbf{t}(\hat{n})\right]_{\hat{s}}
\end{gathered}
$$

where the epoch $\hat{n}$ in Eqs. (17) and (18) is the maximizer of the test statistic $T(n)$ in time, that is $\hat{n}=\arg \max T(n)$, with $n \in\{n \mid T(n)>\gamma\}$, i.e. an estimate of the time-of-arrival of the anomaly.

\section{Simulation Results}

As a proof of the concept, an scenario with $S$ satellites uniformly distributed in azimuth and identical elevation angle of $45^{\circ}$ is proposed. This geometry guarantees an small Dilution of Precision (DOP, [1]). In hypothesis $\mathcal{H}_{1}$, a unite cycle-slip is produced after $N$ samples on the carrier-phase of one satellite chosen randomly. To emulate some slow evolution of the ray geometry in order to force a time-varying eigen-structure, the $s$-th satellite elevation angle is changed at a constant angular speed of $v_{s}$, where $\left\{v_{s}\right\}_{1=1, \ldots, S}$ are independent random variables uniformly distributed within the interval $\left[-v_{\max }, v_{\max }\right]$, and $v_{\max }$ characterizes the overall time-varying nature of the noise subspace to be tracked. For concreteness, for a sampling period of $1 \mathrm{sec}$, a worst-case value of $v_{\max }=0.1^{\circ}$ per sample is assumed, [8], which is almost an order of magnitude larger than the typical values given in real scenarios. The de-trending filter size and order are $K=81$ and $L=4$, respectively.

The goal of the following tests is to demonstrate the performance gain of the multi-channel cycle-slip detection in the presence of high receiver dynamics with respect to classical single channel strategies. To this end, the receiver dynamics are emulated as a circular motion at constant speed of 0.05 turns per sample with a radius of five relative to the unite step-anomaly, such that the de-trending filter is not able to cancel if classical single-channel detection is applied. Every performance indicator value is estimated using $2 \times 10^{5}$ experiments.

Fig. (2) shows the detection performance in terms of the achieved complementary area under ROC (Receiver Operating Characteristics, [11]) (1-AUC). It is seen that single-channel detection performs well only in non-kinematic mode (fixed receiver). The two non-kinematic curves shown in the figure constitute, in fact, a relevant benchmark, i.e. the inherent performance lower bound associated to the cycle-slip detection problem, against which to compare the performance of the proposed technique. The single-channel approach gives too much positives when the receiver has high dynamics, confirming the inability of classical polynomial fitting approaches to cancel receiver dynamics, as they work efficiently only to cancel satellite dynamics. In contrast, the proposed adaptive multichannel approach, which estimates the noise subspace just from the past $N=100$ samples, provides significant discrimination capability between anomalies and receiver dynamics, as appreciated by smaller values achieved of 1-AUC. Moreover, an interesting satellite-diversity phenomenon arises that yields improved performance when the redundancy of the scenario (number of satellites above 4) increases. In other words, the higher the dimension of the noise subspace, the higher the dimension of the equivalent anomaly MIMO channel $\mathbf{C}$ in Eq. (10), and the higher the detection robustness. This diversity effect highly compensates the impact of increased number of nuisance parameters in case of large data dimension. In fact, the penalty caused by dimensionality is only observed in nonkinematic benchmark mode, where the curve of $S=6$ slightly outperforms the curve of $S=14$.

Finally, Fig. (3) aims at showing the ability of the algorithm to correctly identify the fault channel, in terms of error proba- 


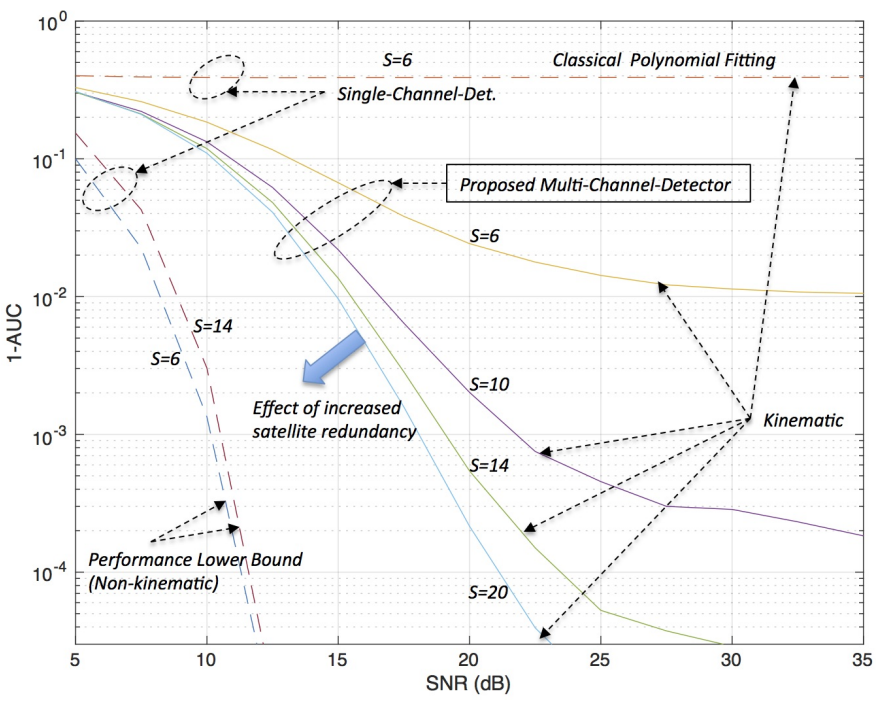

Fig. 2. Complementary area under ROC (1-AUC) of step-anomaly detection as function of SNR, $\sigma^{-2}$, of multi-channel vs. single-channel approaches.

bility of fault channel identification. As it can be appreciated, the error probability decreases quickly as the number of redundant channels increases, exhibiting, once more, a satellite diversity gain effect, specially at moderate SNR. Notice that the typical worst-case values of SNR in GPS are around $20 \mathrm{~dB}$ for low-elevation angles. The impact of $N$ (the number of samples used for estimating the noise subspace of residuals using Eq. (5)) is also shown, which confirms a saturating effect for large $N$ and a robust performance for moderate $N$. Moreover, even with few samples (small values of $N$ ) the subspace is well identified.

\section{CONCLUSIONS}

A multi-channel detector of step-anomalies is presented that makes use of the redundancy of the measurements to cancel common perturbations that are prone to mask their presence. The method provides significant robustness to high receiver dynamics compared with classical single-channel polynomial fitting approaches, avoiding the necessity to accurately tracking them. Canceling the receiver dynamics by subspace estimation provides a notable performance gain in terms of false positives, requires few samples to work efficiently, and allows the use of robust and well-known subspace tracking methods. The maximum benefit is provided in critical receiver dynamics (just when polynomial-fitting approaches clearly fail), as then the dominant major subspace is well identified with few samples. Therefore, tracking the subspace appears to be much more robust than tracking the receiver position when the purpose is canceling the receiver dynamics to avoid false cycleslip detections. With enough redundancy, the performance consistently approaches to the the non-kinematic benchmark bounds and, therefore, the technique shows a potential ability for improving integrity in precise positioning.

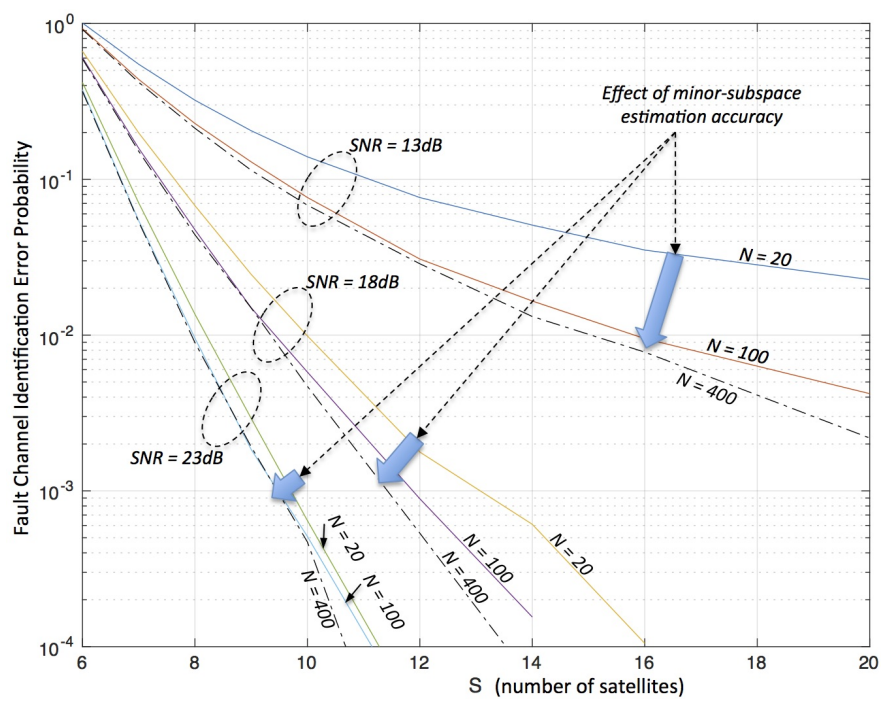

Fig. 3. Fault channel identification error probability in kinematic mode as a function of the multi-channel diversity $(S)$ for different SNR and $N$.

\section{REFERENCES}

[1] J. Sanz, J. M. Juan, and M. Hernández, GNSS Data Processing. ESA Communications, (ESA TM-23/1, May 2013) 2013, vol. 1: Fundamentals and Algorithms.

[2] E. Simona-Lohan, D. A. de Diego, J. A. López-Salcedo, G. SecoGranados, P. Boto, and P. Fernandes, "Unambiguous techniques in modernized GNSS signals," IEEE Signal Process. Magazine, pp. 3852, Sept. 2017.

[3] J. Lee, Y. J. Morton, J. Lee, H.-S. Moon, and J. Seo, "Monitoring and mitigation of ionospheric anomalies for GNSS-based safety critical systems," IEEE Signal Process. Magazine, pp. 96-110, Sept. 2017.

[4] M. Tahir and K. Mazher, "Singular spectrum based smoothing of GNSS pseudorange dynamics," IEEE Communications Letters, vol. 20, no. 8, pp. 1551-1554, August 2016.

[5] P. An, P. Sun, and X. Tang, "Ionospheric scintillation receiving algorithm based on open-loop tracking," in IEEE 3rd Information Technology and Mechatronics Engineering Conference, Oct. 2017, pp. 537-542.

[6] K. Mazher and M. Tahir, "Small cycle slip detection using singular spectrum analysis," in 2016 24th European Signal Processing Conference (EUSIPCO), 2016.

[7] H. Yi, H. Wang, X. Wang, S. Zhang, H. Wang, W. Yang, and S. Wang, "A novel real time cycle slip detection and repair method for a single dual-frequency GNSS receiver," in 2017 Joint Conference of the European Frequency and Time Forum and IEEE International Frequency Control Symposium (EFTF/IFCS), July. 2017, pp. 371-374.

[8] G. Xu, GPS. Theory, Algorithms and Applications. Berlin: SpringerVerlag, 2007.

[9] G. Wübbena, "GPS carrier phases and clock modeling," in GPSTechniques Applied to Geodesy and Surveying. Proceedings of the International GPS-Workshop, Darmstadt, F.R.G. Lecture Notes in Earth Sciences., E. Groten and R. Strauss, Eds., vol. 19, April 1988.

[10] T. F. Fath-Allah, "A new approach for cycle slips repairing using GPS single frequency data," World Appl. Sci. J., vol. 8, no. 3, pp. 315-325, 2010.

[11] S. M. Kay, Fundamentals of Statistical Signal Processing: Detection Theory. New York: Prentice-Hall, 1998, vol. 2.

[12] M. Kaddour, N. A. Tmazirte, M. E. El, Z. Naja, and N. Moubayed, "Fault detection and exclusion of cycle slips for carrier-phase in GNSS positionning," in 18th Int. Conf. on Inf. Fusion, Sept. 2015, pp. 593-598.

[13] Y. Qin, B. Li, and L. Lou, "Multi-frequency BeiDou cycle slip and data gap repair with geometry-based model," in 2017 Forum on Cooperative Positioning and Service (CPGP), May. 2017, pp. 251-257.

[14] G. Zhang, Q. Gui, and S. Han, "A bayesian method of GNSS cycle slips detection based on ARMA model," in 2017 Forum on Cooperative Positioning and Service (CPGP), Oct. 2017, pp. 219-222.

[15] K. Abed-Meraim, S. Attallah, A. Chkeif, and Y. Hua, "Orthogonal Oja Algorithm," IEEE Signal Process. Letters, vol. 7, no. 5, pp. 116-119, May. 2000. 